\title{
Determination of Collagen
}

\section{Fibers Arrangement in Bone Tissue by Using Transformations of Raman Spectra Maps}

\author{
Tomasz Buchwald, Marek Kozielski, and Mirosław Szybowicz \\ Faculty of Technical Physics, Poznan University of Technology, Nieszawska 13a, 60-965 Poznań, Poland \\ Correspondence should be addressed to Tomasz Buchwald, scabby@ poczta.onet.pl
}

Copyright (C) 2012 Tomasz Buchwald et al. This is an open access article distributed under the Creative Commons Attribution License, which permits unrestricted use, distribution, and reproduction in any medium, provided the original work is properly cited.

\begin{abstract}
The goal of this work was to evaluate the ability of Raman spectroscopy to identify molecular organization and chemical composition of extracellular matrix such as the collagen fibers arrangement, the level of mineralization, and the carbonate accumulation in mineral phase in spongy bone of the human head of the femur. Changes in composition and structure of the spongy bone tissue were illustrated using maps of polarized Raman spectra. In particular, the purpose of the present study was determination of arrangement of mineralized collagen on surface of trabecula by using transformations of Raman spectra maps. Transformations of Raman spectra maps were needed in order to remove impact of chemical composition on images of Raman spectra map, which display the collagen fibers orientation. These transformations allow to obtain simultaneously the distribution of constituents of bone and arrangement of collagen fibers on tissue surface. A method to indicate the collagen orientations is developed to understand the molecular organization in healthy and unhealthy bone at the microstructural level.
\end{abstract}

Keywords: Raman spectroscopy, bone tissue, collagen fibers arrangement

\section{Introduction}

Bone tissue is a heterogeneous material composed of cells such as osteoblasts, osteocytes, osteoclasts, and an extracellular matrix, which consists primarily of collagen fibrils, mineral phase (hydroxyapatite and carbonate apatite crystals), and water [1-3]. The organic matrix is mainly made up of type I collagen fibers [4]. The collagen fibers are more elastic and resistant to fractures than the apatite crystals. Therefore, the bone structure strength is determined by the mineral components [5], whereas the elasticity of the tissue is determined by the organic components [6]. By combining the high toughness of collagen and the high stiffness of mineral, bone achieves its remarkable mechanical properties [7]. Bone in human and other mammal bodies is generally classified into two different forms: cortical bone and spongy bone. Cortical bone, also known as compact bone, is found mainly in the shaft of long bones and forms the outer shell around spongy bone. Spongy bone, also known as cancellous or trabecular bone, is found in the end of long bones, in vertebrae and in flat bones $[8,9]$. Cortical bone is much denser and less porous than spongy bone. Both bone types have a hierarchical structure. The basic first level structure of cortical 
bone is osteon (osseous laminae arranged in a concentric pattern around Haversian canals), whereas spongy bone is formed by a network of trabeculae of different shape and thickness arranged along the directions of action of mechanical pressure forces $[10,11]$. The mode of trabeculae arrangement allows to distribute and dissipate the energy from articular contact loads.

Bone mass or bone mineral density is the most common parameter to clinically assess the bone quality. However, the content of mineral and organic constituents, the spongy bone microarchitecture, the orientation and cross-linking of collagen component, the accumulation of microdamage, and the bone remodeling activity or turnover also influence on the bone quality [3, 12-16]. In this way, assessment of the bone quality is complicated. One approach to address this issue is Raman microspectroscopy. This vibrational spectroscopy has been successfully applied to characterize tissues, especially bone tissue [17-20]. The use of Raman spectroscopy in biological investigation offers several advantages. This physical technique allows to determine the chemical composition and structure of tissue at the microstructural level $[21,22]$. Raman spectroscopy offers micron-scale spatial resolution $(0.5-1 \mu \mathrm{m})$ and enables analysis of biologically important sites such as individual cement lines, individual lamellae, regions around microcracks, and human dentin tubules [23, 24]. Spectroscopic data are obtained in a noninvasive manner, so the same sample can be examined by the various analytical methods. Raman spectra are not obscured by water, allowing analysis of fully hydrated samples, with minimal sample preparation. The visible or near-infrared optics, that are used by Raman instruments, are compatible with fiber-optic probes used in clinical instruments such as arthroscopes. In this way, Raman spectroscopy method could be used directly in arthroscopic surgery. Esmonde-White et al. showed the use of Raman spectroscopy for arthroscopy of human joint tissues using a custom-built fiber-optic probe [25]. Raman spectroscopy can provide detailed chemical information on the mineral and collagen matrix components in unprocessed bone preparations [20, 26]. Raman bands are sensitive to the molecular orientation; therefore the arrangement of collagen fibers and apatite crystals in osteons and trabeculae can be also determined [16, 27-29]. By using a motorized microscope stage, Raman spectroscopy technique allows to obtain the Raman spectra maps of the sample giving spatial information and showing the distribution and orientation of the chemical constituents on tissue surface. In earlier work, Raman spectral imaging has been used to study the complex organized systems such as bone tissue [24, 27, 28, 30].

Polarization of excited and Raman scattered light provides the additional information on the structure and orientation of the molecular components of bone. Some of bands in Raman spectrum of bone tissue are quite sensitive to the molecular orientation [27-29]. Spectral parameters of phosphate $\nu_{1}$ and amide I Raman bands change more in different polarization direction of excited and scattered beam than spectral parameters of amide III, phosphate $\nu_{2}$, and phosphate $\nu_{4}$ Raman bands. The crystallographic Caxis of apatite crystals is along the collagen fibers and is associated with $\nu_{1}$ phosphate vibrations. Thus the phosphate $\nu_{1}$ band intensity has a maximum value when the polarization of incident light is parallel to the orientation of collagen fibers [31]. The collagen triple helix structure determines the positions of the amide bonds with respect to the backbone. The $\mathrm{C}=\mathrm{O}$ bonds in amide $\mathrm{I}$ are perpendicular to the collagen molecular axis; therefore the amide $\mathrm{I}$ band, which is associated with the $\mathrm{C}=\mathrm{O}$ stretching vibration, is more intense in the polarization direction perpendicular to the collagen fiber axis [32]. In this way, the phosphate $\nu_{1}$ and amide I bands are quite sensitive to the polarization direction of the incident and scattered light and have been related to the local orientation of collagen fibers and apatite crystals in the bone tissue. On the other hand, the bands such as those assigned to amide III, phosphate $\nu_{2}$, and phosphate $\nu_{4}$ are less sensitive to the orientation effects and indicate changes in mineral and organic 
composition of bone tissue [29]. In this study the linearly polarized incident and scattered light was used to obtain Raman spectra maps, which allow to show collagen fibers arrangement on trabecula surface.

Raman spectra provide information on both mineral and organic matrix composition in bone tissue. Two of the most commonly reported measurements of Raman analysis of bone chemistry is the hydroxyapatite to collagen ratio and the carbonate apatite to hydroxyapatite ratio, obtained from the integrated areas of the phosphate $\nu_{2}$ and $\nu_{4}$, carbonate $\nu_{1}$, and amide III bands. These parameters provide an indication of mineralization of bone tissue and describe the degree of carbonate substitution in the apatite crystal lattice. Raman spectroscopy also allows to indicate the bone tissue organization such as orientation of collagen fibers in osteons and trabeculae by the use of hydroxyapatite to collagen ratio parameter. The hydroxyapatite to collagen ratio is obtained from integrated area of the phosphate $\nu_{1}$ and amide I bands, which are dependent on tissue organization. In earlier studies the proposed parameters have been used to estimate the compositional and structural properties of bone tissue [16, 23, 24, 33-36]. Irregularity of surface of biological material influences intensity of bands in Raman spectrum. Due to potential variations in distance from the objective to the sample, the band area ratios were employed to describe composition and structure of bone tissue.

The goal of this work was to evaluate the ability of Raman spectroscopy to identify molecular organization and chemical composition of extracellular matrix such as the collagen fibers arrangement, the level of mineralization, and the carbonate accumulation in mineral phase in spongy bone of the human head of the femur. Changes in composition and structure of the spongy bone tissue were illustrated using maps of polarized Raman spectra. In particular, the purpose of the present study was determination of arrangement of mineralized collagen on surface of trabecula by using transformations of Raman spectra maps. Transformations of Raman spectra maps, new application to determine the bone properties, were needed in order to remove impact of chemical composition on images of Raman spectra map, which display the collagen fibers orientation. These transformations allow to obtain simultaneously the distribution of constituents of bone and arrangement of collagen fibers on tissue surface. A method to indicate the collagen orientations is developed to understand the molecular organization in healthy and unhealthy bone at the microstructural level.

\section{Materials and Method}

In this study, Raman microspectroscopy was used to estimate the chemical composition and orientation of collagen fibers of human spongy bone of femur heads collected from the five patients between 60 and 85 years of age, who underwent a high-energy femoral neck fracture. The treatment of choice in the above-mentioned patients was hip replacement surgery (total hip arthroplasty). The cross-sections of five millimeters in thickness were obtained from heads of femurs of these patients. In this work, the results of a study on one representative femur head from a 63-year-old woman are presented. The study has got the approval of the local Bioethical Commission at the Wielkopolska Medical Chamber in Poznań (no 14/2008 from August 27, 2008).

All the spectroscopic measurements were performed on confocal Raman microspectroscope (inVia) made by Renishaw equipped with diode pumped laser emitting $785 \mathrm{~nm}$ infrared wavelength. The laser beam was tightly focused on the sample surface through a Leica 50x LWD microscope objective (LWD: long working distance) with numerical aperture (NA) equals 0.5 , leading to a laser beam diameter 


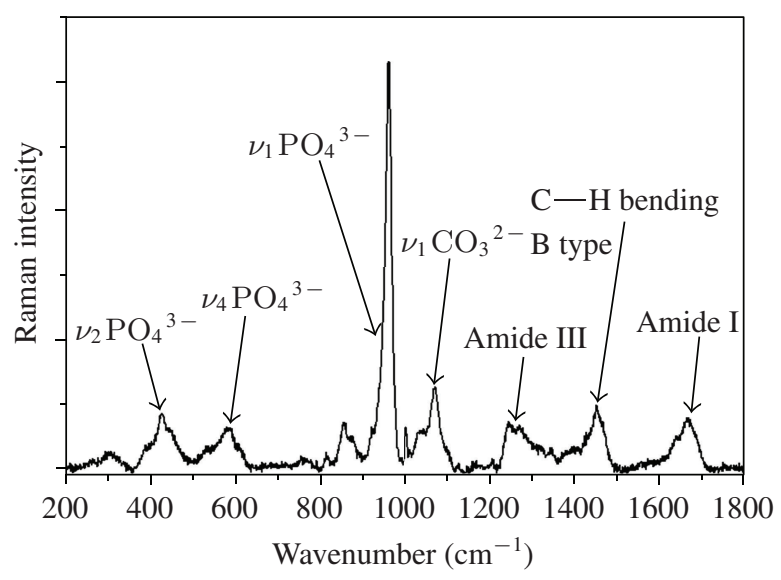

Figure 1: Representative Raman spectrum collected from spongy bone tissue showing the major bands and the corresponding compounds. Background signal has been removed.

about $2 \mu \mathrm{m}$. The Raman scattering spectra were acquired from surface of the bone tissue in the backscattering geometry in the spectral range of $200-1800 \mathrm{~cm}^{-1}$. During measurements, to minimize sample degradation or sample heating an incident power was dimmed to approximately $10 \mathrm{~mW}$. The wavenumber and intensity were regularly calibrated with the use of $521 \mathrm{~cm}^{-1}$ band of a silicon internal sample. The position of the microscope objective with respect to the bone tissue was piezoelectrically controlled in the three axes during surface mapping. The measurements were made under a microscope in the high confocality mode with the depth resolution equals $2.2 \mu \mathrm{m}$. The incident light was linearly polarized optionally with a half-wave plate. The Raman scattered light was detected in the linear polarization (vertical and horizontal direction) using polarisers. The Raman spectra at different laser polarizations were obtained on the same position for given sample. The Raman spectra maps were achieved collecting single spectra, with an exposure time of $10 \mathrm{~s}$ for each spectrum without accumulation. The Raman images of surface of bone tissue were acquired in the rectangular areas of $100 \times 100 \mu \mathrm{m}^{2}$ with steps of $10 \mu \mathrm{m}$ at seven different sites of particular femur heads (thirty-five different sites of five femur heads). The analyses of arrangement of mineralized collagen on surface of trabecula by using transformations of these thirty-five Raman maps gave the same results. Therefore, the results of a study on one site of femur head are presented. The samples were measured at room temperature $(296 \mathrm{~K})$. Cosmic ray artefacts were removed and analyses of the spectra were performed in the WIRE 3.1 (Renishaw) software. Rayleigh scattering background was subtracted manually from each raw spectrum by using the polynomial curve. The integrated areas of Raman bands were calculated by curve-fitting or deconvolution in single spectra. The integrated area ratios of Raman bands were used to detect differences in the composition and structure of bone tissue. The images were generated by the OriginPro 8.0 software.

\section{Results and Discussion}

Figure 1 gives a general view of the Raman spectra from spongy bone sample showing the major bands corresponding to the mineral and organic phases. The position of the main vibrational bands in the observed Raman spectra of bone tissue and their respective assignments are listed in Table 1 . The mineral 
Table 1: Position of the main vibrational bands in the observed Raman spectra of bone tissue and their respective assignments.

\begin{tabular}{lc}
\hline Wavenumber $\left(\mathrm{cm}^{-1}\right)$ & Assignment \\
\hline 431 & $\nu_{2}\left(\mathrm{PO}_{4}{ }^{3-}\right)$ \\
589 & $\nu_{4}\left(\mathrm{PO}_{4}{ }^{3-}\right)$ \\
961 & $\nu_{1}\left(\mathrm{PO}_{4}{ }^{3-}\right)$ \\
1072 & $\nu_{1}\left(\mathrm{CO}_{3}{ }^{2-}\right) \mathrm{B}$ type \\
1103 & $\nu\left(\mathrm{CO}_{3}{ }^{2-}\right)$ A type \\
$1200-1305$ & Amide III \\
$1405-1490$ & $\mathrm{C}-\mathrm{H}$ bending \\
$1599-1701$ & Amide I \\
$2800-3100$ & $\mathrm{C}-\mathrm{H}$ stretching \\
\hline
\end{tabular}

bands of the spectrum are dominated by the $\nu_{1}$ phosphate $\left(\mathrm{PO}_{4}{ }^{3-}\right)$ internal mode at $961 \mathrm{~cm}^{-1}$. The other markers of the mineral component connected with the $\nu_{2}$ and $\nu_{4}$ phosphate vibrations are detected at $431 \mathrm{~cm}^{-1}$ and $589 \mathrm{~cm}^{-1}$, respectively [20,27]. The internal modes of the carbonate group $\left(\mathrm{CO}_{3}{ }^{2-}\right)$ are detected at $1072 \mathrm{~cm}^{-1}$ ( $\nu_{1}$ mode of B-type carbonate) and $1103 \mathrm{~cm}^{-1}$ (mode of A-type carbonate) [16]. However, the weakness of the A-type carbonate band does not permit getting information about the composition of bone tissue; therefore B-type mode only is used. The bands assigned to the organic components (collagen and noncollagen moieties) are found in regions: $\sim 1200-1305 \mathrm{~cm}^{-1}$ (amide III), $\sim 1599-1701 \mathrm{~cm}^{-1}$ (amide I), $1405-1490 \mathrm{~cm}^{-1}$, and $\sim 2800-3100 \mathrm{~cm}^{-1}$ (bending and stretching modes of $\mathrm{C}-\mathrm{H}$ groups, resp.) [20, 28]. The Raman signal depends not only on the composition but also on the local orientation of collagen fibers or apatite crystals. Therefore, to indicate changes in chemical composition in bone tissue bands, which are less sensitive to the orientation effects were used. The mineral and organic composition were determined from the following Raman bands: the amide III (integrated area $\sim 1200-1305 \mathrm{~cm}^{-1}$ ), phosphate $\nu_{2}$ (integrated area $\sim 370-486 \mathrm{~cm}^{-1}$ ), phosphate $\nu_{4}$ (integrated area $\sim 511-632 \mathrm{~cm}^{-1}$ ), and carbonate $\nu_{1}$ (integrated area $\sim 1041-1107 \mathrm{~cm}^{-1}$ ). Dependency of amide I and phosphate $\nu_{2}$ Raman bands intensity on structural organization allows to determine arrangement of collagen fibers in bone tissue. The orientation of collagen fibers was indicated using the integrated area $\sim 1599-1701 \mathrm{~cm}^{-1}$ of amide I band and $\sim 932-989 \mathrm{~cm}^{-1}$ of phosphate $\nu_{2}$ band.

The ratios of integrated area of the appropriate bands in the Raman spectra were employed to obtain the biochemical properties of bone tissue. As markers of chemical composition the ratios of $\nu_{2} \mathrm{PO}_{4}{ }^{3-}$ /amide III, $\nu_{4} \mathrm{PO}_{4}{ }^{3-}$ /amide III, and $\nu_{1} \mathrm{CO}_{3}{ }^{2-} / \nu_{2} \mathrm{PO}_{4}{ }^{3-}$ were used. The phosphate to collagen ratio identified differences in mineralization of bone tissue, whereas the carbonate-to-phosphate ratio gave information about the degree of carbonate accumulation in the apatite crystal lattice. The ratio of $\nu_{1} \mathrm{PO}_{4}{ }^{3-}$ /amide I was calculated and used to investigate orientation of collagen fibers. The compositional and structural changes in spongy bone were illustrated using maps of polarized Raman spectra. Figure 2 presents images of the ratios of $\nu_{2} \mathrm{PO}_{4}{ }^{3-}$ /amide III (Figures 2(a), 2(d), and 2(g)), $\nu_{1} \mathrm{CO}_{3}{ }^{2-} / \nu_{2} \mathrm{PO}_{4}{ }^{3-}$ (Figures 2(b), 2(e), and 2(h)) and $\nu_{1} \mathrm{PO}_{4}{ }^{3-}$ /amide I (Figures 2(c), 2(f), and 2(i)). The maps of Raman spectra were generated in the vertical and horizontal polarization of incident and scattered light. The arrows in the figures indicate these polarization directions. Each image displays the same area of spongy 


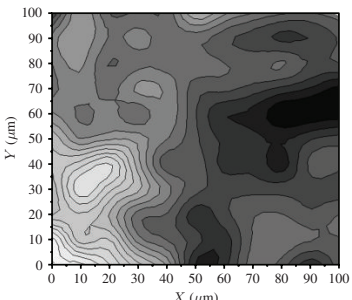

(a)

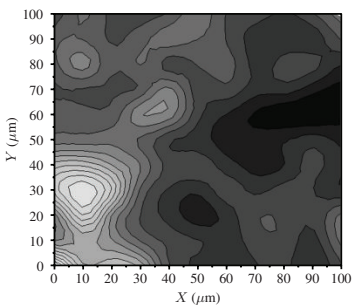

(d)

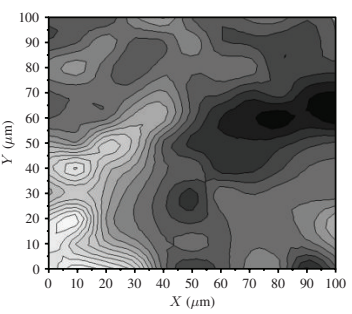

(g)

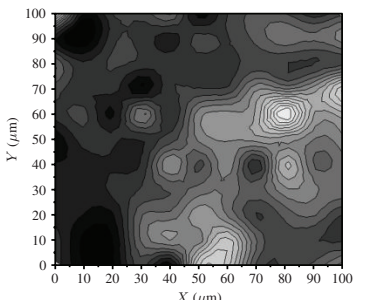

(b)

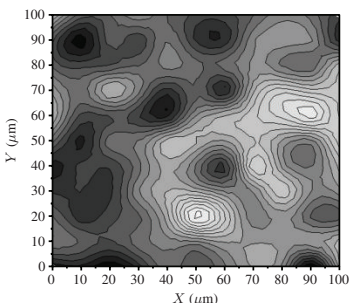

(e)

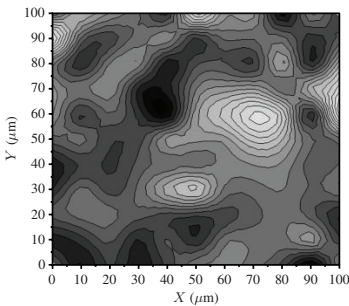

(h)

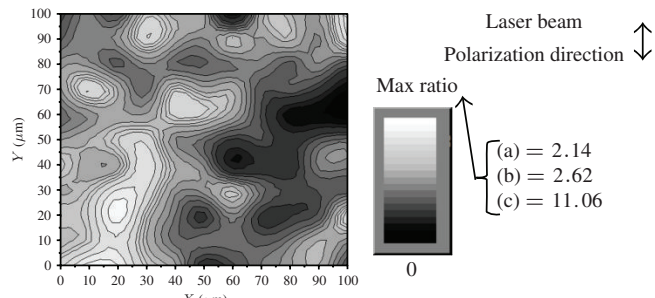

(c)
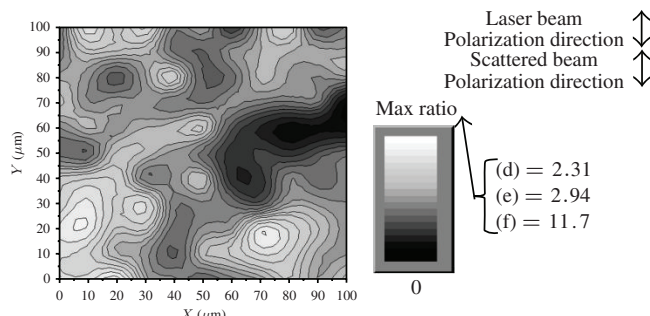

(f)
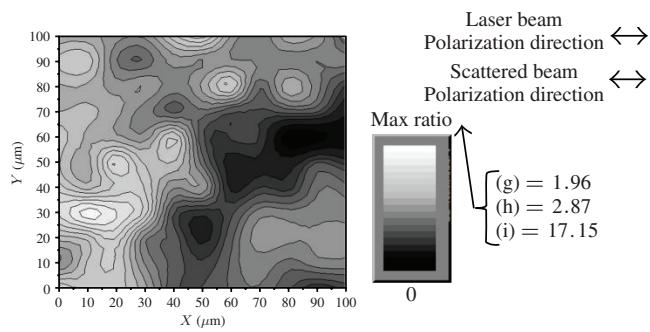

(i)

Figure 2: The contrast images based on the ratios of $\nu_{2} \mathrm{PO}_{4}{ }^{3-} /$ amide III ((a), (b), and (g)), $\nu_{1} \mathrm{CO}_{3}{ }^{2-} / \nu_{2} \mathrm{PO}_{4}{ }^{3-}\left((\mathrm{b}),(\mathrm{e})\right.$, and (h)), and $\nu_{1} \mathrm{PO}_{4}{ }^{3-}$ /amide I ((c), (f), (i)). Arrows indicate polarization of laser incident and scattered beams, and the color bar displays the maximum ratio for each image.

bone surface. In the scale bars, the maximum values of the ratios are specified and the minimum value is 0 for all images. The bright contrast corresponds to the maximum ratio, while dark to the lowest one.

The bands used in the ratios of $\nu_{2} \mathrm{PO}_{4}{ }^{3-}$ /amide III and $\nu_{1} \mathrm{CO}_{3}{ }^{2-} / \nu_{2} \mathrm{PO}_{4}{ }^{3-}$ are less sensitive to orientation effect, so the maps based on these ratios are similar and do not show considerable changes in the contrast for different polarizations of incident and scattered light. In this way, the $\nu_{2} \mathrm{PO}_{4}{ }^{3-}$ /amide III and $\nu_{1} \mathrm{CO}_{3}{ }^{2-} / \nu_{2} \mathrm{PO}_{4}{ }^{3-}$ ratio images are used as an indicator of distribution of chemical components in spongy bone tissue. Conclusions on the degree of mineralization of spongy bone can be drawn from Figure 2 showing the ratios of $\nu_{2} \mathrm{PO}_{4}{ }^{3-}$ /amide III obtained for the vertical polarization of laser light (V polarization) (Figure 2(a)), the vertical polarization of laser incident and scattered beams (VV polarization) (Figure 2(d)), and the horizontal polarization of laser incident and scattered beams (HH polarization) (Figure 2(g)). These images present very similar contrast changes despite the fact that the Raman maps were obtained in different polarization directions. Therefore, contrast images based on the ratio of $\nu_{2} \mathrm{PO}_{4}{ }^{3-}$ /amide III are a good indicator of local variations in the content of hydroxyapatite crystals with respect to that of collagen on surface of trabecula. The brighter and darker areas in these images correspond to higher and lower ratios of mineral to organic content. Figure 2 displays images 
for the ratios of $\nu_{1} \mathrm{CO}_{3}{ }^{2-} / \nu_{2} \mathrm{PO}_{4}{ }^{3-}$ obtained for the vertical polarization of laser light (Figure 2(b)), the vertical polarization of laser incident and scattered beams (Figure 2(e)), and the horizontal polarization of laser incident and scattered beams (Figure 2(h)). These images illustrating relative distribution of mineral components on the surface of trabecula present also similar contrast changes as those for the ratios of $\nu_{2} \mathrm{PO}_{4}{ }^{3-}$ /amide III despite the fact that the Raman maps were obtained in different polarization directions. The brighter and darker areas in these images correspond to higher and lower ratios of carbonate apatite to hydroxyapatite content. The higher ratio of carbonate-to-phosphate crystals in Figures 2(b), 2(e), and 2(h) occurs in the same area as the lower ratio of hydroxyapatite to collagen content in Figures 2(a), 2(d), and 2(g). It is possible that the increased carbonate substitution in the apatite crystal lattice occurred in response to deficient mineralization.

Figure 2 displays $\nu_{1} \mathrm{PO}_{4}{ }^{3-}$ /amide I ratio contrast images of the bands quite sensitive to the collagen fibers orientation obtained for the vertical polarization of laser light (Figure 2(c)), the vertical polarization of laser incident and scattered beams (Figure 2(f)), and the horizontal polarization of laser incident and scattered beams (Figure 2(i)). The $\nu_{1} \mathrm{PO}_{4}{ }^{3-}$ /amide I ratio contrast images should determine the collagen fibers arrangement in trabecula; however these images do not show a pronounced structural effect. Figures 2(c), 2(f), and 2(i) based on the $\nu_{1} \mathrm{PO}_{4}{ }^{3-}$ /amide I ratio reveal slight contrast changes in comparison with those in the other ratio images referring to the chemical composition in trabecula (Figures 2(a), 2(d), and 2(g)). The similarity in the character of the maps referring to the collagen orientation and chemical composition is probably related to the distribution of bone tissue constituents. This means that differences in contrast in the maps referring to chemical composition are too large with respect to these referring to collagen fibers arrangement, so the orientation effect is undetectable. Hence, Figures 2(c), 2(f), and 2(i) present very similar contrast images to Figures 2(a), 2(d), and 2(g). Moreover, the images in Figures 2(f) and 2(i) illustrating collagen orientation on surface of trabecula are almost identical and their contrasts do not change much for different laser incident and scattered light polarizations. Therefore, it is difficult to conclude if the highest contrast corresponds to the orientation of collagen fibers or to the changes in chemical composition. To remove impact of chemical composition on $\nu_{1} \mathrm{PO}_{4}{ }^{3-}$ /amide I ratio contrast images obtained for different laser incident and scattered light polarizations, transformations of these contrast images were used. Transformations permit to display on images the orientation of collagen fibers without chemical composition.

Figure 3 displays contrast images obtained by using transformations of Raman spectra maps. In the scale bars, the maximum values of the ratios are specified and the minimum value is 0 for all images. The bright contrast corresponds to the maximum values, while dark to the lowest one. Figures 3(a), and 3(b) display images obtained by using the first transformation of Raman spectra maps. Figure 3(a) illustrates image with different values between $\nu_{1} \mathrm{PO}_{4}{ }^{3-}$ /amide I ratio values obtained for the horizontal polarization of laser incident and scattered beams ( $\mathrm{HH}$ polarization) and $\nu_{1} \mathrm{PO}_{4}{ }^{3-}$ /amide I ratio values obtained only for the horizontal polarization of laser light (H polarization). Figure 3(b) illustrates image with difference values between $\nu_{1} \mathrm{PO}_{4}{ }^{3-}$ /amide I ratio values obtained for the vertical polarization of laser incident and scattered beams (VV polarization) and $\nu_{1} \mathrm{PO}_{4}{ }^{3-}$ /amide I ratio values obtained only for the vertical polarization of laser light (V polarization). This first transformation of Raman spectra maps allows to display collagen fibers orientation, which is parallel to the horizontal direction (Figure 3(a)) and parallel to the vertical direction (Figure 3(b)). If the orientation of collagen fibers is not parallel to the horizontal or vertical polarization of laser light, so if the fibers are skewed, then the changes in polarizability of collagen molecule are detected for both light polarizations. Therefore, this collagen 


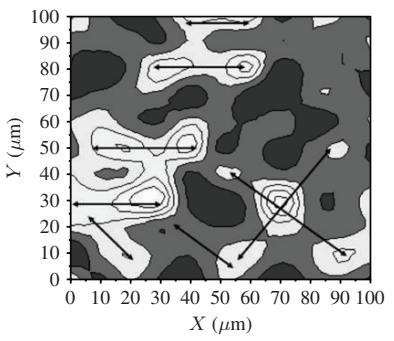

(a)

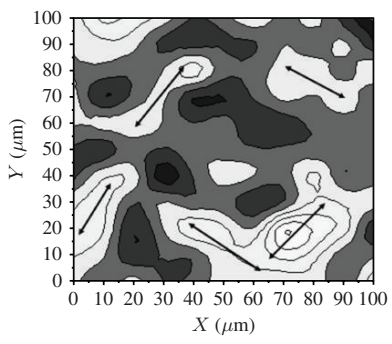

(b)

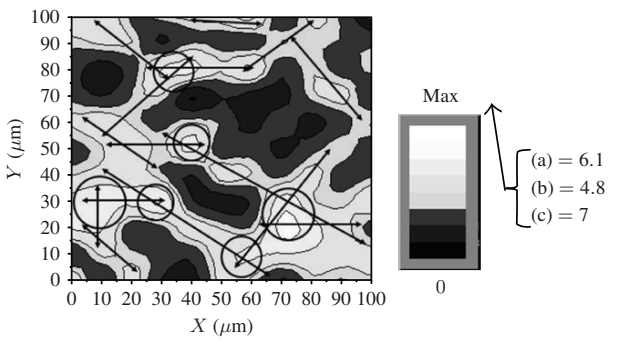

(c)

Figure 3: The contrast images obtained by using transformations of Raman spectra maps. The contrast images with difference values between $\nu_{1} \mathrm{PO}_{4}{ }^{3-}$ /amide I ratio values obtained for the $\mathrm{HH}$ polarization and $\mathrm{H}$ polarization $[\mathrm{HH}-\mathrm{H}](\mathrm{a})$, for the $\mathrm{VV}$ polarization and $\mathrm{V}$ polarization $[\mathrm{VV}-\mathrm{V}]$ (b). The contrast images with the total values $[(\mathrm{VV}-\mathrm{V})+(\mathrm{HH}-\mathrm{H})]$, which is the sum of $[\mathrm{VV}-\mathrm{V}]$ values and $[\mathrm{HH}-\mathrm{H}]$ values from the first transformation (c). Arrows indicate collagen fibers arrangement on surface of trabecula, and the color bar displays the maximum value for each image.

fibers arrangement is also visible simultaneously in Figures 3(a) and 3(b). The arrows in Figures 3(a) and 3(b) indicate collagen fibers arrangement on surface of trabecula.

Figure 3(c) displays contrast image obtained by using the second transformation of Raman spectra maps. Figure $3(\mathrm{c})$ illustrates image with the total values $[(\mathrm{VV}-\mathrm{V})+(\mathrm{HH}-\mathrm{H})]$ which is the sum of $[\mathrm{VV}-\mathrm{V}]$ values and $[\mathrm{HH}-\mathrm{H}]$ values from the first transformation. This second transformation allows to display collagen fibers arrangement on surface of trabecula in each direction. Circles in Figure 3(c) indicate areas with the maximum values. Areas with the maximum values are found in the parts of trabecula surface where the arrows are crossed. It could mean that collagen fibers overlap each other in these parts of trabecula. The results of Raman maps transformations show that this method to analysis of collagen fibers arrangement on surface of trabecula could be very useful to determine properties of bone tissue at microstructural level.

\section{Conclusion}

In this study the linearly polarized incident and scattered light was used to obtain the Raman spectra maps to show changes in the structure of trabecula. The Raman spectra maps based on the ratios of $\nu_{2} \mathrm{PO}_{4}{ }^{3-}$ /amide III and $\nu_{1} \mathrm{CO}_{3}{ }^{2-} / \nu_{2} \mathrm{PO}_{4}{ }^{3-}$ do not show considerable changes for different polarizations of incident and scattered light, so these images were taken as an indicator of spongy bone composition, that is, give the information about relative amount of hydroxyapatite to collagen and carbonate apatite to hydroxyapatite. The Raman spectra maps based on the ratio of $\nu_{1} \mathrm{PO}_{4}{ }^{3-}$ /amide I should show the collagen fibers arrangement in trabecula, because bands in this ratio are quite sensitive to the collagen orientation. However, this image does not show distinct changes for different polarizations of incident and scattered light. It could mean that differences in contrast in the maps referring to chemical composition are too large with respect to those referring to collagen fibers orientation so the orientation effect is undetectable. These results suggest to use transformations of Raman spectra maps, which allow to remove impact of chemical composition on contrast images of these maps. In this study, we use two transformations of Raman spectra maps. The first transformation removed impact of chemical composition 
on contrast images and indicated collagen fibers arrangement on surface of trabecula in parallel to the horizontal and vertical direction. The second transformation allowed to display collagen fibers arrangement on surface of trabecula in each direction. The results allow concluding that $\nu_{1} \mathrm{PO}_{4}{ }^{3-}$ /amide I ratios permit to determine the orientations of collagen fibers in spongy bone tissue; however if we would like to say more about collagen fibers arrangement, we could use transformations proposed in this work.

Results of investigations demonstrate the possibility of the Raman spectroscopy application for microanalysis of composition and collagen fibers arrangement in the human bone tissue. The method, which allows to distinguish between orientation and compositional contribution, is crucial as bone is comprised of mineralized collagen fibers with alternating orientation in osteons and trabeculae. Understanding the bone tissue organization at the microstructural level can help finding the origins of bone diseases such as osteoporosis or osteoarthritis. Determination of the mode of collagen fibers arrangement permits evaluation of the biomechanical properties of bone, so provides information of susceptibility of bone to fracture.

\section{Acknowledgments}

The samples were prepared at the Poznan University of Medical Sciences. All the measurements were carried out on Renishaw inVia microscope at the Poznan University of Technology. All experimental procedures were approved by the local bioethical commission at the Wielkopolska Medical Chamber in Poznan (no. 14/2008 from August 27, 2008). The study was performed within the research project DS PB 64-001/12 of the Poznan University of Technology. The author Tomasz Buchwald declares that he is a scholarship holder within the project "Scholarship support for Ph.D. students specializing in majors strategic for Wielkopolska's development", Submeasure 8.2.2 Human Capital Operational Programme, cofinanced by European Union under the European Social Fund. The authors are much grateful to dr n. med. B. Ciesielczyk and dr. n. med. A. Piotrowski from the University of Medical Sciences for preparation of the material to be studied.

\section{References}

[1] A. L. Boskey, "Bone mineralization," in Bone Biomechanics, S. C. Cowin, Ed., pp. 5.1-5.34, CRC Press, Boca Raton, Fla, USA, 3rd edition, 2001.

[2] S. I. Harada and G. A. Rodan, "Control of osteoblast function and regulation of bone mass," Nature, vol. 423, no. 6937, pp. 349-355, 2003.

[3] S. Weiner and H. D. Wagner, "The material bone: structure-mechanical function relations," Annual Review of Materials Science, vol. 28, no. 1, pp. 271-298, 1998.

[4] J. Sierpowska, M. J. Lammi, M. A. Hakulinen, J. S. Jurvelin, R. Lappalainen, and J. Töyräs, "Effect of human trabecular bone composition on its electrical properties," Medical Engineering and Physics, vol. 29, no. 8, pp. 845-852, 2007.

[5] G. Boivin and P. J. Meunier, "Effects of bisphosphonates on matrix mineralization," Journal of Musculoskeletal Neuronal Interactions, vol. 2, no. 6, pp. 538-543, 2002.

[6] M. J. Favus, Primer on the Metabolic Bone Diseases and Dsorders of Mineral Metabolism, American Society for Bone and Mineral Research, Washington, DC, USA, 5th edition, 2003.

[7] O. De Carmejane, M. D. Morris, M. K. Davis et al., "Bone chemical structure response to mechanical stress studied by high pressure Raman spectroscopy," Calcified Tissue International, vol. 76, no. 3, pp. 207-213, 2005. 
[8] M. Centrella, T. L. McCarthy, and E. Canalis, "Skeletal tissue and transforming growth factor $\beta$," FASEB Journal, vol. 2, no. 15, pp. 3066-3073, 1988.

[9] S. C. Cowin, Bone Mechanics Handbook, CRC Press, Boca Raton, Fla, USA, 2001.

[10] J. A. Buckwalter, M. J. Glimcher, R. R. Cooper, and R. Recker, "Bone biology. Part I: structure, blood supply, cells, matrix, and mineralization," Journal of Bone and Joint Surgery-Series A, vol. 77, no. 8, pp. 1256-1275, 1995.

[11] J. A. Buckwalter, M. J. Glimcher, R. R. Cooper, and R. Recker, "Bone biology. Part II. Formation form, modeling, remodeling, and regulation of cell function," Journal of Bone and Joint SurgerySeries A, vol. 77, no. 8, pp. 1276-1289, 1995.

[12] B. R. McCreadie, M. D. Morris, T. C. Chen et al., "Bone tissue compositional differences in women with and without osteoporotic fracture," Bone, vol. 39, no. 6, pp. 1190-1195, 2006.

[13] E. Seeman and P. D. Delmas, "Bone quality - the material and structural basis of bone strength and fragility," The New England Journal of Medicine, vol. 354, no. 21, pp. 2250-2261, 2006.

[14] M. D. Morris and G. S. Mandair, "Raman assessment of bone quality," Clinical Orthopaedics and Related Research, vol. 469, no. 8, pp. 2160-2169, 2011.

[15] P. Fratzl, H. S. Gupta, E. P. Paschalis, and P. Roschger, "Structure and mechanical quality of the collagen-mineral nano-composite in bone," Journal of Materials Chemistry, vol. 14, no. 14, pp. 21152123, 2004.

[16] S. Gamsjaeger, A. Masic, P. Roschger et al., "Cortical bone composition and orientation as a function of animal and tissue age in mice by Raman spectroscopy," Bone, vol. 47, no. 2, pp. 392399, 2010.

[17] J. S. Nyman, A. J. Makowski, C. A. Patil et al., "Measuring differences in compositional properties of bone tissue by confocal raman spectroscopy," Calcified Tissue International, vol. 89, no. 2, pp. $111-122,2011$.

[18] M. D. Morris, "Raman spectroscopy of bone and cartilage," in Emerging Raman Applications and Techniques in Biomedical and Pharmaceutical Fields, Biological and Medical Physics, Biomedical Engineering, pp. 347-364, Springer, Berlin, Germany, 2010.

[19] M. D. Morris, M. V. Schulmerich, K. A. Dooley, and K. A. Esmonde-White, "Vibrational spectroscopic imaging of hard tissues," in Infrared and Raman Spectroscopic Imaging, R. Salzer and H. W. Siesler, Eds., Wiley-VCH Verlag GmbH \& Co. KGaA, Weinheim, Germany, 2009.

[20] X. Bi, C. A. Patil, C. C. Lynch, G. M. Pharr, A. Mahadevan-Jansen, and J. S. Nyman, "Raman and mechanical properties correlate at whole bone- and tissue-levels in a genetic mouse model," Journal of Biomechanics, vol. 44, no. 2, pp. 297-303, 2011.

[21] G. Penel, C. Delfosse, M. Descamps, and G. Leroy, "Composition of bone and apatitic biomaterials as revealed by intravital Raman microspectroscopy," Bone, vol. 36, no. 5, pp. 893-901, 2005.

[22] M. Raghavan, N. D. Sahar, R. H. Wilson et al., "Quantitative polarized Raman spectroscopy in highly turbid bone tissue," Journal of Biomedical Optics, vol. 15, no. 3, Article ID 037001, 2010.

[23] J. A. Timlin, A. Carden, and M. D. Morris, "Chemical microstructure of cortical bone probed by Raman transects," Applied Spectroscopy, vol. 53, no. 11, pp. 1429-1435, 1999.

[24] J. A. Timlin, A. Carden, M. D. Morris et al., "Spatial distribution of phosphate species in mature and newly generated mammalian bone by hyperspectral Raman imaging," Journal of Biomedical Optics, vol. 4, no. 1, pp. 28-34, 1999.

[25] K. A. Esmonde-White, F. W. L. Esmonde-White, M. D. Morris, and B. J. Roessler, "Fiber-optic Raman spectroscopy of joint tissues," Analyst, vol. 136, no. 8, pp. 1675-1685, 2011.

[26] Y. Iwasaki, J. J. Kazama, H. Yamato, and M. Fukagawa, "Changes in chemical composition of cortical bone associated with bone fragility in rat model with chronic kidney disease," Bone, vol. 48, no. 6, pp. 1260-1267, 2011.

[27] M. Kazanci, H. D. Wagner, N. I. Manjubala et al., "Raman imaging of two orthogonal planes within cortical bone," Bone, vol. 41, no. 3, pp. 456-461, 2007. 
[28] M. Kazanci, P. Roschger, E. P. Paschalis, K. Klaushofer, and P. Fratzl, "Bone osteonal tissues by Raman spectral mapping: orientation-composition," Journal of Structural Biology, vol. 156, no. 3, pp. 489-496, 2006.

[29] M. Kozielski, T. Buchwald, M. Szybowicz, Z. Błaszczak, A. Piotrowski, and B. Ciesielczyk, "Determination of composition and structure of spongy bone tissue in human head of femur by Raman spectral mapping," Journal of Materials Science, vol. 22, pp. 1653-1661, 2011.

[30] C. P. Tarnowski, M. A. Ignelzi, W. Wang, J. M. Taboas, S. A. Goldstein, and M. D. Morris, "Earliest mineral and matrix changes in force-induced musculoskeletal disease as revealed by Raman microspectroscopic imaging," Journal of Bone and Mineral Research, vol. 19, no. 1, pp. 64-71, 2004.

[31] H. Tsuda and J. Arends, "Orientational micro-Raman spectroscopy on hydroxyapatite single crystals and human enamel crystallites," Journal of Dental Research, vol. 73, no. 11, pp. 1703$1710,1994$.

[32] M. Tsuboi, J. M. Benevides, P. Bondre, and G. J. Thomas Jr., "Structural details of the thermophilic filamentous bacteriophage PH75 determined by polarized Raman microspectroscopy," Biochemistry, vol. 44, no. 12, pp. 4861-4869, 2005.

[33] A. Carden, R. M. Rajachar, M. D. Morris, and D. H. Kohn, "Ultrastructural changes accompanying the mechanical deformation of bone tissue: a Raman imaging study," Calcified Tissue International, vol. 72, no. 2, pp. 166-175, 2003.

[34] C. P. Tarnowski, M. A. Ignelzi, and M. D. Morris, "Mineralization of developing mouse calvaria as revealed by raman microspectroscopy," Journal of Bone and Mineral Research, vol. 17, no. 6, pp. 1118-1126, 2002.

[35] K. A. Dehring, N. J. Crane, A. R. Smukler, J. B. McHugh, B. J. Roessler, and M. D. Morris, "Identifying chemical changes in subchondral bone taken from murine knee joints using raman spectroscopy," Applied Spectroscopy, vol. 60, no. 10, pp. 1134-1141, 2006.

[36] T. Buchwald, K. Niciejewski, M. Kozielski, M. Szybowicz, M. Siatkowski, and H. Krauss, "Identifying compositional and structural changes in spongy and subchondral bone from the hip joints of patients with osteoarthritis using Raman spectroscopy," Journal of Biomedical Optics, vol. 17, Article ID 017007, 2012. 


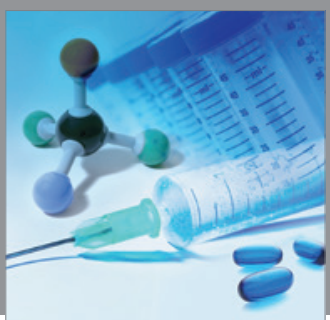

International Journal of

Medicinal Chemistry

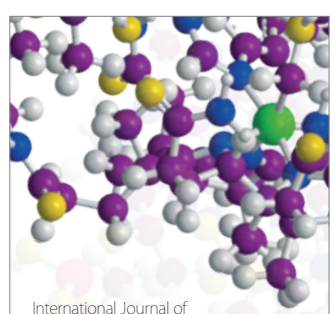

Carbohydrate Chemistry

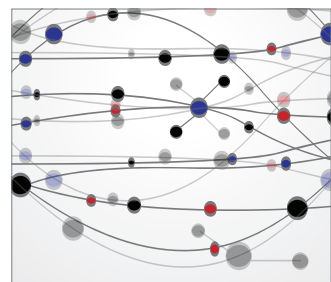

The Scientific World Journal
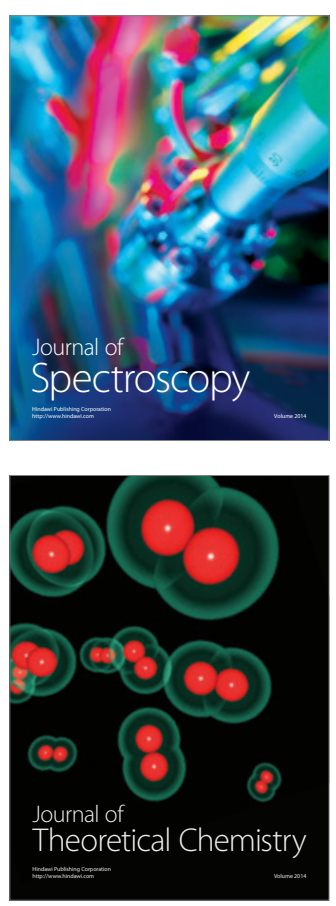
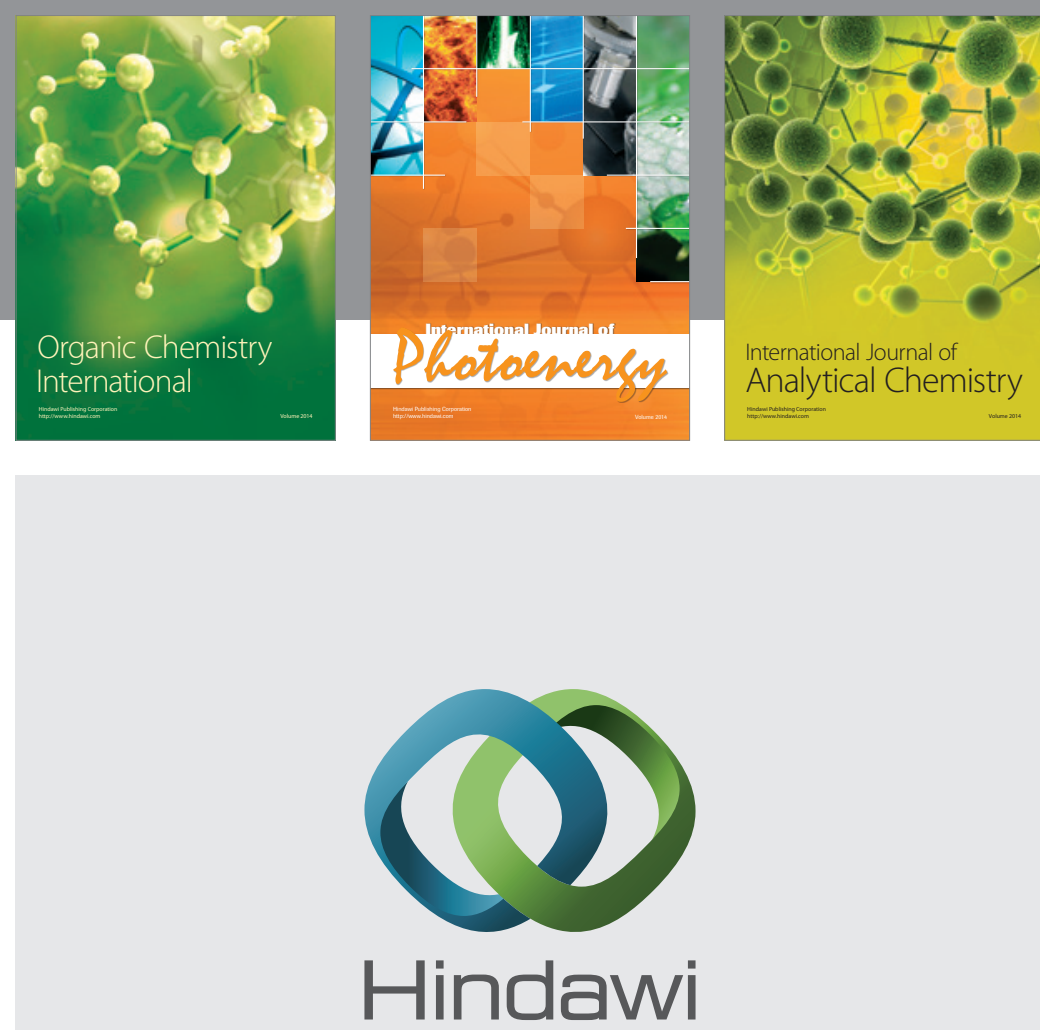

Submit your manuscripts at

http://www.hindawi.com
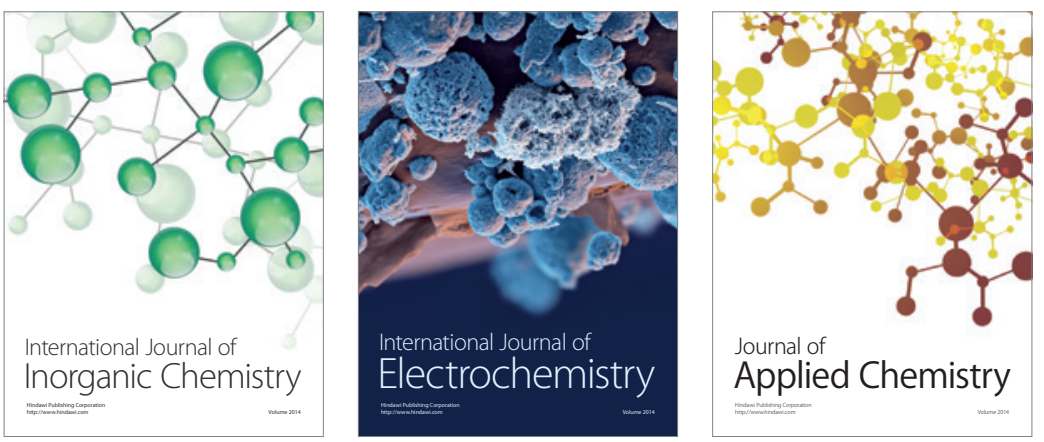

Journal of

Applied Chemistry
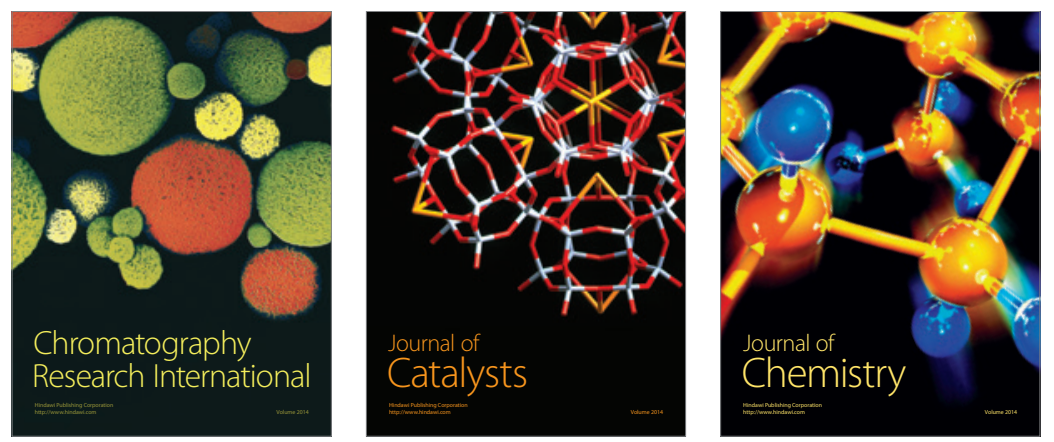
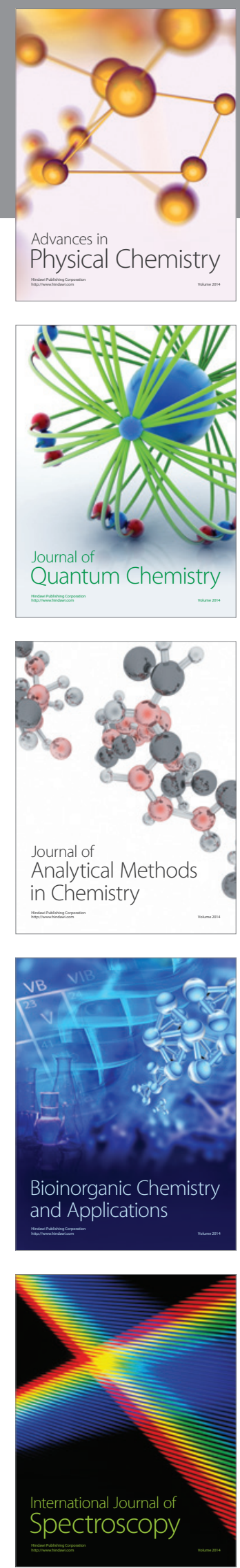